\title{
Robust passification via static output feedback - LMI results
}

\author{
Dimitri Peaucelle, Alexander Fradkov* and Boris Andrievsky* \\ LAAS-CNRS \\ 7 avenue du colonel Roche, 31077 Toulouse, FRANCE \\ Tel. 0561336309 fax: 0561336969 \\ email: \{ peaucelle \}@laas.fr
}

10th May 2005

\begin{abstract}
Non-conservative LMI conditions of robust strict G-passification are presented and a design method is given for static output-feedback control. An application example to robust controller design for a cruise missile model is given.

Keywords: Linear systems, robust control, passivity, passification, LMI, static output-feedback
\end{abstract}

\section{INTRODUCTION}

Passivity-based techniques play more and more important role both in linear and nonlinear control due to simplicity and physical meaning of design and good robustness properties of the resulting controllers $[3,20,15,16]$. One of the most

*Institute for Problems of Mechanical Engineering of RAS, 61 Bolshoy av. V.O., St Petersburg, 199178, Russia. email: \{ alf , andr \}@ control.ipme.ru 
powerful passivity-based design methods is passification (sometimes called passivation) - finding a state or output feedback rendering the closed loop system passive $[21,6,13]$. Among applications of passification approach are adaptive control [1]; control of partially linear composite systems [19, 13], flight control $[12,8]$, process control $[25,9]$.

An important question for application of passivity-based approach is to find feasibility conditions of the method, i.e. passifiability conditions. In the case of linear MIMO systems such conditions were found in [5]: a proper transfer function $W(s)$ can be rendered strictly positive real (SPR) by means of static output feedback if and only if it is hyper-minimum-phase (HMP) - minimumphase with symmetric and positive definite high frequency gain. Obviously, HMP is also necessary and sufficient condition of output feedback strict passification (see below). Further references on passification can be found in [6].

In view of uncertainty inevitably appearing in real life problems, a lot of efforts were put recently to obtain robust solutions to control problems. Surprisingly, the problem of robust passification did not draw much attention yet. Existing conditions of robust passifiability either are only sufficient (conservative) [12] or apply only to special cases, important for signal processing, in particular to systems with zero relative degree [24]. Note that most works on related problem of robust SPR systems design are also devoted to systems with zero relative degree, see $[23,2,10]$ and references therein.

Therefore, it is of interest to obtain necessary and sufficient conditions of robust passifiability and to develop techniques of robust passification for linear proper MIMO systems. This is just the goal of the present paper. In fact in the paper a more general problem of $G$-passification of non-square systems is studied, where $G$ is a rectangular matrix squaring down the initial system. The proposed conditions and design technique heavily rely on the methodology of Linear Matrix Inequalities (LMI) and using appropriate software.

In the Section 2 the problem statement and some preliminaries are given. In view of above mentioned equivalence between passifiability and hyper-minimumphaseness, first, the problem of LMI interpretation of HMP and G-HMP conditions is studied. Conditions for a matrix $G$ to ensure HMP property of the transfer function $G W(s)$ are established in terms of appropriate LMIs in Section 3. In Section 4 , a result concerning design of a robustly passifying output feedback 
is given. An example of application of the previous results to robust controller design for cruise missile is considered in Section 5.

\section{Preliminaries and problem statement}

Notations: $\mathbb{R}^{m \times n}$ and $\mathbb{C}^{m \times n}$ are the sets of $m$-by- $n$ real and complex matrices respectively. $A^{T}$ is the transpose of the matrix $A$ and $A^{*}$ is its transpose conjugate. $A^{\perp}$ is a full rank matrix whose columns span the null-space of $A$. The columns of $A^{\circ}$ form an orthogonal basis of $A^{*}$, i.e. $\left[\begin{array}{ll}A^{\perp} & A^{\circ}\end{array}\right]$ is full rank. $\mathbb{1}$ and $\mathbb{O}$ are respectively the identity and the zero matrices of appropriate dimensions. For Hermitian matrices, $A>(\geq) B$ if and only if $A-B$ is positive (semi) definite. The notation $\left[\begin{array}{ll}A & * \\ B & C\end{array}\right]$ stands for the Hermitian matrix $\left[\begin{array}{cc}A & B^{*} \\ B & C\end{array}\right]$.

Consider the following uncertain system

$$
\left\{\begin{array}{l}
\dot{x}=A(\Delta) x+B u \\
y=C x
\end{array}\right.
$$

where $x=x(t) \in \mathbb{C}^{n}, u=u(t) \in \mathbb{C}^{m}, y=y(t) \in \mathbb{C}^{l}$ are state, input and output vectors, respectively. $A, B, C$ are matrices of appropriate size, $B$ being of full rank. Define the matrix $N=B^{* \perp}$ whose columns span the null-space of $B^{*}$. Define $W(s, \Delta)$ to be the uncertain transfer matrix of the system such that

$$
W(s, \Delta)=C(s \mathbb{1}-A(\Delta))^{-1} B
$$

and assume $A(\Delta)$ is rational with respect to $\Delta$

$$
A(\Delta)=A+B_{\Delta} \Delta\left(\mathbb{1}-D_{\Delta} \Delta\right)^{-1} C_{\Delta}
$$

where $\Delta$ is a constant norm-bounded unstructured uncertainty that belongs to the set

$$
\Delta=\left\{\Delta \in \mathbb{C}^{m_{\Delta} \times l_{\Delta}}: \Delta^{*} \Delta \leq \mathbb{1}\right\} .
$$

The model is said to be a Linear Fractional Transform (LFT), build as the feedback connection of the uncertain matrix $\left(w_{\Delta}=\Delta z_{\Delta}\right)$ with the linear system

$$
\left\{\begin{array}{l}
\dot{x}=A x+B_{\Delta} w_{\Delta}+B u \\
z_{\Delta}=C_{\Delta} x+D_{\Delta} w_{\Delta} \\
y=C x
\end{array}\right.
$$


Special case $D_{\Delta}=\mathbb{O}$ corresponds to uncertainty linearly entering some coefficients of the model $\left(A(\Delta)=A+B_{\Delta} \Delta C_{\Delta}\right)$. The LFT is assumed to be wellposed, that is $\left(\mathbb{1}-D_{\Delta} \Delta\right)$ is non-singular for all admissible uncertainties $\Delta \in \mathbb{\Delta}$ and $A(\Delta)$ is in a bounded set.

The case with real-valued variables and parameters $\left(x=x(t) \in \mathbb{R}^{n}, u=\right.$ $u(t) \in \mathbb{R}^{m}, y=y(t) \in \mathbb{R}^{l}$ ) will be called real case while the case of complexvalued variables and parameters will be called complex case.

Let $G$ be a prespecified $m \times l$-matrix. Extending the definitions of [6] to uncertain systems, we define here the concepts manipulated in the paper and formulate the considered static-output feedback design specifications.

Definition 1 System (1) is called robustly strictly G-passive if for any $\Delta \in \mathbb{\Delta}$ there exists a quadratic function $V(x, \Delta)=x^{*} H(\Delta) x$ (storage function) with a positive definite $n \times n$-matrix $H(\Delta)=H^{*}(\Delta)>\mathbb{0}$ and a scalar $\rho(\Delta)>0$ such that

$$
\begin{aligned}
x^{*}(t) H(\Delta) & x(t) \leq x^{*}(0) H(\Delta) x(0) \\
& +\int_{0}^{t}\left[u(\theta)^{*} G y(\theta)-\rho(\Delta)|x(\theta)|^{2}\right] d \theta
\end{aligned}
$$

holds for any solution of the system (1).

Obviously, if $l=m$ and $G=\mathbb{1}$ is identity matrix, then $G$-passivity coincides with conventional passivity property. Moreover, for a given $G$, the modified system $G W(s)$ is passive if and only if the original system $W(s)$ is $G$-passive. But $G$-passification of $W(s)$ (i.e. finding $K$ such that $G W(s)(\mathbb{1}-K W(s))^{-1}$ is passive) is not equivalent to passification of $G W(s)$. These two problems coincide only under the assumption of finding a square $\bar{K}$ such that $K=\bar{K} G$. This "squaring down" procedure changes the number of unknowns which may be important to preserve, e.g. for adaptive control, where reducing the number of adjustable parameters may decrease transient performance of adaptive systems, see [1]. To summarize, $G$-passification of $W(s, \Delta)$ is not equivalent to passification of $G W(s, \Delta)$, thus justifying the terminology.

Definition 2 System (1) is called uniformly robustly strictly G-passive if for any $\Delta \in \Delta$ there exists a unique quadratic function $V(x)=x^{*} H x$ (storage function) with $H=H^{*}>\mathbb{O}$ and a unique scalar $\rho>0$ such that (3) holds for all uncertainties $\Delta \in \mathbb{A}$ and for any solution of the system (1). 
Trivially the uniform robsut strict $G$-passivity is conservative for robust strict $G$ passivity.

In this paper we consider three static output-feedback passification problems defined as follows.

Problem 1 Find a parameter-dependent $m \times l$-matrix $K(\Delta)$ such that the system (1) with the output feedback

$$
u=K(\Delta) y+v
$$

where $v \in \mathbb{C}^{m}$ is new input, is robustly strictly $G$-passive.

Problem 2 Find a single $m \times l$-matrix $K(\Delta)=K$ such that the system (1) with the output feedback (4) is robustly strictly G-passive.

Problem 3 Find a single $m \times$ l-matrix $K(\Delta)=K$ such that the system (1) with the output feedback (4) is uniformly robustly strictly G-passive.

The paper gives non conservative LMI conditions for the existence of a solution for all three problems and Problem 3 is associated with an LMI design method.

Definition 3 The system (1) is called robustly G hyper minimum phase (G-HMP) if for all uncertainties $\Delta \in \triangle$ the polynomial

$$
\varphi(s, \Delta)=\operatorname{det}(s \mathbb{1}-A(\Delta)) \operatorname{det} G W(s, \Delta)
$$

is Hurwitz (its zeros belong to the open left half-plane) and the high-frequency gain of $G W(s, \Delta)$ is a square symmetric positive definite matrix

$$
G C B=B^{*} C^{*} G^{*}>\mathbb{0}
$$

The following theorem providing solvability conditions for the robust $G$-passification problem is a direct consequence of the results of $[5,6]$.

Theorem 1 The three following conditions are equivalent:

i) The system (1) is robustly G-hyper minimum phase,

ii) There is a (parameter-dependent) solution to Problem 1. 
iii) There is a (unique over all uncertainties) solution to Problem 2.

Proof: Equivalence between $i$ ) and ii) is directly obtained when applying [6, Theorem 2] for each value of $\Delta$. Then note that due to [6, Corollary 3] one can always take $K(\Delta)=-k(\Delta) G$ as parameter-dependent solutions to Problem 1. Moreover if $\tilde{k}(\Delta) \geq k(\Delta)$, then $\tilde{K}(\Delta)=-\tilde{k}(\Delta) G$ is also a solution to Problem 1. Due to the well-posedness of the LFT we get that $\max _{\Delta \in \Delta} k(\Delta)=k_{*}$ is finite and $K_{*}=-k_{*} G$ is a solution to Problem 2. Therefore ii) implies iii) and the converse is trivial.

The next section is devoted to LMI results for robust $G$-HMP analysis, based on these and on Theorem 1 the results therefore give LMI conditions for the existence of a solutions to Problems 1 and 2. In Section 4, LMI methods are then given to solve problem 3 .

\section{Robust $G$-HMP}

Theorem $2 W(s, \Delta), \Delta \in \triangle$ is robustly $G$-HMP if and only if there exists a solution $P \in \mathbb{C}$ to the LMI constraints (6) and

$$
\begin{gathered}
P>\mathbb{0} \\
{\left[\begin{array}{cc}
P N^{*} M A N+N^{*} A^{*} M N P & P N^{*} M B_{\Delta} \\
B_{\Delta}^{*} M N P & -\mathbb{1}
\end{array}\right]} \\
+\left[\begin{array}{c}
N^{*} C_{\Delta}^{*} \\
D_{\Delta}^{*}
\end{array}\right]\left[\begin{array}{ll}
C_{\Delta} N & D_{\Delta}
\end{array}\right]<\mathbb{0}
\end{gathered}
$$

where $N=(G C)^{\perp}$ and $M=\left(N N^{*}+B B^{*}\right)^{-1}$. In the real case, $P$ can be chosen real valued.

Proof: First note that

$$
\begin{gathered}
\varphi(s, \Delta)=\operatorname{det} \Sigma(s, \Delta) \\
\Sigma(s, \Delta)=\left[\begin{array}{cc}
s \mathbb{1}-A(\Delta) & -B \\
G C & 0
\end{array}\right] .
\end{gathered}
$$


$\varphi(s, \Delta) \neq 0$ is therefore equivalent to assessing that $\Sigma(s, \Delta)$ is non singular. Therefore we can write that $\varphi(s, \Delta)$ is Hurwitz for all $\Delta \in \Delta$ if and only if $\Sigma(s, \Delta)$ is non singular for all $\Delta \in \Delta$ and all $s \in \mathbb{C}^{+}$. That is that the only solution to

$$
\Sigma(s, \Delta)\left(\begin{array}{c}
w \\
\bar{z}
\end{array}\right)=0
$$

is the zero vector $\left(w^{*} \bar{z}^{*}\right)=0$. Define $N=(G C)^{\perp}$, the problem reduces to prove that the only solution to

$$
\begin{aligned}
& N z_{N}-B \bar{z}-A N w_{N}-B_{\Delta} w_{\Delta}=0 \\
& z_{\Delta}-C_{\Delta} N w_{N}-D_{\Delta} w_{\Delta}=0 \\
& w_{N}=s^{-1} z_{N} \\
& w_{\Delta}=\Delta z_{\Delta}
\end{aligned}
$$

is the zero vector $\left(z_{N}^{*} z_{\Delta}^{*} \bar{z}^{*} w_{N}^{*} w_{\Delta}^{*}\right)=0$. In turn this is equivalent to assessing well-posedness of the following feedback system

$$
\begin{aligned}
& \left(\begin{array}{c}
w_{N} \\
w_{\Delta}
\end{array}\right)=\underbrace{\left[\begin{array}{ccc}
s^{-1} \mathbb{1} & 0 & 0 \\
\mathbb{0} & \Delta & 0
\end{array}\right]}_{\nabla}\left(\begin{array}{c}
z_{N} \\
z_{\Delta} \\
\bar{z}
\end{array}\right): \begin{array}{c}
s^{-1} \in \mathbb{C}^{+} \\
\Delta \in \Delta
\end{array} \\
& \underbrace{\left[\begin{array}{ccc}
N & 0 & -B \\
0 & \mathbb{1} & 0
\end{array}\right]}_{\mathcal{E}}\left(\begin{array}{c}
z_{N} \\
z_{\Delta} \\
\bar{z}
\end{array}\right)=\underbrace{\left[\begin{array}{cc}
A N & B_{\Delta} \\
C_{\Delta} N & D_{\Delta}
\end{array}\right]}_{\mathcal{A}}\left(\begin{array}{c}
w \\
w_{\Delta}
\end{array}\right) .
\end{aligned}
$$

The $\mathcal{E}$ matrix proves to have

$$
\mathcal{E}^{\circ}=\left[\begin{array}{cc}
N^{*} & 0 \\
0 & \mathbb{1} \\
-B^{*} & 0
\end{array}\right] .
$$

Applying results of Theorem 1 in [18] with

$$
\mathcal{Q}=\left[\begin{array}{ll}
\mathcal{E} \mathcal{E}^{\circ} & -\mathcal{A}
\end{array}\right]^{\perp}=\left[\begin{array}{cc}
M A N & M B_{\Delta} \\
C_{\Delta} N & D_{\Delta} \\
\mathbb{1} & \mathbb{0} \\
0 & \mathbb{1}
\end{array}\right]
$$


where $N=(G C)^{\perp}$ and $M=\left(N N^{*}+B B^{*}\right)^{-1}$, implies without conservatism that there exist a Hermitian matrix $\Theta$ (can be taken real-valued in the real case) such that for all $s^{-1} \in \mathbb{C}^{+}$and $\Delta \in \Delta$

$$
\mathcal{Q}^{*} \Theta \mathcal{Q}>\mathbb{0}, \quad\left[\begin{array}{ll}
\mathbb{1} & \mathcal{E}^{\circ *} \nabla^{*}
\end{array}\right] \Theta\left[\begin{array}{c}
\mathbb{1} \\
\nabla \mathcal{E}^{\circ}
\end{array}\right] \leq \mathbb{0}
$$

For the given uncertain operator

$$
\nabla \mathcal{E}^{\circ}=\left[\begin{array}{cc}
s^{-1} N^{\prime} & \mathbb{0} \\
\mathbb{0} & \Delta
\end{array}\right]: \begin{aligned}
s^{-1} & \in \mathbb{C}^{+} \\
\Delta & \in \mathbb{\Delta}
\end{aligned}
$$

the separator $\Theta$ can be chosen without conservatism such that

$$
\Theta=\left[\begin{array}{cccc}
\mathbb{0} & \mathbb{0} & N \hat{P} & \mathbb{0} \\
\mathbb{0} & \tau \mathbb{1} & \mathbb{0} & \mathbb{0} \\
\hat{P} N^{\prime} & \mathbb{0} & \mathbb{0} & \mathbb{0} \\
\mathbb{0} & \mathbb{0} & \mathbb{0} & -\tau \mathbb{1}
\end{array}\right]
$$

where $\hat{P}$ is positive definite and $\tau$ is a positive scalar. The remaining condition $\mathcal{Q}^{*} \Theta \mathcal{Q}>\mathbb{O}$ is exactly (7) when taking $P=\tau^{-1} \hat{P}$.

At our knowledge the result of Theorem 2 is new, even for the case of LTI systems without uncertainties. In that case the LMIs formulae are slightly less involved. Define $W(s) \sim(A, B, C)$ a system without uncertainties, a direct corollary of the previous theorem is as follows.

Corollary $1 W(s)$ is $G$-HMP if and only if there exists a solution $P \in \mathbb{C}$ to the LMI constraints (6) and

$$
\begin{gathered}
P>\mathbb{0} \\
P N^{*} M A N+N^{*} A^{*} M N P<\mathbb{0}
\end{gathered}
$$

where $N=(G C)^{\perp}$ and $M=\left(N N^{*}+B B^{*}\right)^{-1}$. In the real case, $P$ can be taken real-valued.

Combining Theorems 1 and 2 we can conclude that Problems 1 and 2 have a solution if and only if the LMI constraints (6) and (7) are satisfied. This existence condition is unfortunately not constructive. Explicit design of a feedback control for the Problem 3 is given in the next section. 


\section{Robust $G$-passification}

Theorem $3 W(s, \Delta)$ is uniformly robustly strictly $G$-passifiable via static outputfeedback if and only if there exists a solution $H \in \mathbb{C}^{n \times n}, K \in \mathbb{C}^{m \times l}$ to the LMI constraints

$$
\begin{gathered}
H>\mathbb{O}, H B=C^{*} G^{*} \\
{\left[\begin{array}{cc}
H A+A^{*} H+ & C^{*}\left(G^{*} K+K^{*} G\right) C \\
B_{\Delta}^{*} H & H B_{\Delta} \\
-\mathbb{1}
\end{array}\right]} \\
+\left[\begin{array}{c}
C_{\Delta}^{*} \\
D_{\Delta}^{*}
\end{array}\right]\left[\begin{array}{ll}
C_{\Delta} & D_{\Delta}
\end{array}\right]<\mathbb{0} .
\end{gathered}
$$

The solution $K$ is such that (4) solves Problem 3.

Proof: Denote $A(\Delta, K)=A(\Delta)+B K C$ the matrix such that the closed-loop system writes:

$$
\dot{x}=A(\Delta, K) x+B v, y=C x
$$

For a given $\Delta$ and $K$, the uniform robust strict $G$-passivity of this system is equivalent according to [6] to the constraints:

$$
\begin{gathered}
H A(\Delta, K)+A^{*}(\Delta, K) H<\mathbb{0} \\
H>\mathbb{0}, H B=C^{*} G^{*}
\end{gathered}
$$

The first two constraints correspond exactly to quadratic stability (in the conventional sense in robust control introduced by [11]) of the system $\dot{x}=A(\Delta, K) x$ which is equivalent [17] to the existence of a symetric positive definite matrix $H>\mathbb{O}$ such that

$$
\begin{aligned}
& {\left[\begin{array}{cc}
H A(K)+A^{*}(K) H & H B_{\Delta} \\
B_{\Delta}^{*} H & -\mathbb{1}
\end{array}\right]} \\
& +\left[\begin{array}{c}
C_{\Delta}^{*} \\
D_{\Delta}^{*}
\end{array}\right]\left[\begin{array}{ll}
C_{\Delta} & D_{\Delta}
\end{array}\right]<\mathbb{0}
\end{aligned}
$$

where $A(K)=A+B K C$. Under these conditions the matrix $H$ is indeed a solution to the two first constraints in (10). To conclude the proof, note that the constraint $H B=C^{*} G^{*}$ implies $H A(K)=H A+C^{*} G^{*} K C$. 
Remark. A most interesting problem is to design simultaneously $G$ and $K$ so as to render the closed-loop system $G$-passive. This problem, as most static-output feedback design problems, is not convex and deserves more attention left for a future paper. One approach would be as in [4] to add a conservative constraint that assumes the existence of a non singular square matrix $M$ such that $H B=$ $B M$. If this linear constraint can be fulfilled, one may take $C^{*} G^{*} K=B S$ which makes the problem of finding $G$ and $K$ purely LMI and the static-feedback is reconstructed taking $K=M^{-1} S$.

Corollary $2 W(s)$ is strictly G-passifiable via static output-feedback if and only if there exists a solution $H \in \mathbb{C}^{n \times n}, K \in \mathbb{C}^{m \times l}$ to the LMI constraints

$$
\begin{gathered}
H>\mathbb{0}, H B=C^{*} G^{*} \\
H A+A^{*} H+C^{*}\left(G^{*} K+K^{*} G\right) C<\mathbb{0} .
\end{gathered}
$$

\section{Example}

Consider the linearized fourth-order model of lateral dynamics for cruise missile including model of actuator dynamics, presented in [7]. Assume that the uncertainty in the model is caused by variations in the flight altitude $h$. Let the measured plant output $y(t)$ be a vector of the yaw angle $\varphi(t)$, yaw angular rate $r(t)$ and the rudder deflection angle $\delta_{r}(t): y(t)=\left[\psi, r, \delta_{r}\right]^{*}$. The control input of the plant is the rudder servo command signal, i.e. $n=4, m=1, l=3$.

Parameters of the state-space model (1) in this case are as follows:

$$
\begin{gathered}
A=\left[\begin{array}{cccc}
0 & 1 & 0 & 0 \\
0 & 0 & 1 & 0 \\
0 & 12 & -0.6 & 5.0 \\
0 & 0 & 0 & -20
\end{array}\right], B=\left[\begin{array}{c}
0 \\
0 \\
0 \\
20
\end{array}\right], C=\left[\begin{array}{llll}
1 & 2 & 0 & 0 \\
0 & 1 & 2 & 0 \\
0 & 0 & 0 & 1
\end{array}\right], \\
B_{\Delta}=\left[\begin{array}{l}
0 \\
0 \\
1 \\
0
\end{array}\right], C_{\Delta}=\left[\begin{array}{llll}
0 & -7.5 & 0.7 & -4.5
\end{array}\right], D_{\Delta}=0 .
\end{gathered}
$$


Note that for this data some coefficients of the matrix $A(\Delta)$ vary in an order of magnitude.

The problem of $G$-passifying the system with a static feedback is considered for a given value of $G$. A more general problem would be to find simultaneously a matrix $G$ and a feedback $K$, it is left for prospective work. Therefore we do not explicit here how the $G$ matrix is selected.

Take $G=\left[\begin{array}{lll}8 & 3 & 1\end{array}\right]$ we then get that

- Inequalities of Theorem 2 have a solution, the system is therefore robustly $G$-HMP. There exists a static feedback that makes the system robustly strictly $G$-passive.

- Inequalities of Theorem 3 have a solution, the system is uniformly strictly $G$-passifiable by static feedback.

Several solutions may then be exhibited.

- First, solve the LMI without any additional constraint:

$$
K_{1}=-\left[\begin{array}{lll}
79.28 & 50.34 & 11.92
\end{array}\right] .
$$

- Second, look for a solution with minimal norm. To do so add the LMI constraint

$$
\left[\begin{array}{ll}
\alpha \mathbb{1} & K \\
K^{\prime} & \mathbb{1}
\end{array}\right]>0
$$

A Schur complement argument shows that this implies $K K^{\prime} \leq \alpha \mathbb{1}$. Minimising $\alpha$ implies to minimise the norm of the control gain and therefore to get a less demanding control in terms of amplification. When applied to the example we get

$$
K_{2}=-\left[\begin{array}{lll}
60.75 & 34.47 & 10.67
\end{array}\right] .
$$

- Third, one may seek a control gain proportional to the $G$ matrix as suggested in $[5,6]$. To do so simply add the constraint $K=-\gamma G$ and eventually minimise $\gamma$. The solution is then

$$
K_{3}=-\left[\begin{array}{lll}
118.53 & 44.45 & 14.82
\end{array}\right] .
$$


To further analyse the results, some simulations are performed for the feedback system obtained with the gain $K_{2}$. They demonstrate good performance of the designed closed-loop system. The time histories of the yaw angle $\psi$ and the rudder angle $\delta_{r}$ for different flight altitudes $h=0.1,5,9 \mathrm{~km}$ are depicted in Fig. 1. The corresponding Nyquist plots for the closed-loop system are shown in Fig. 2 .
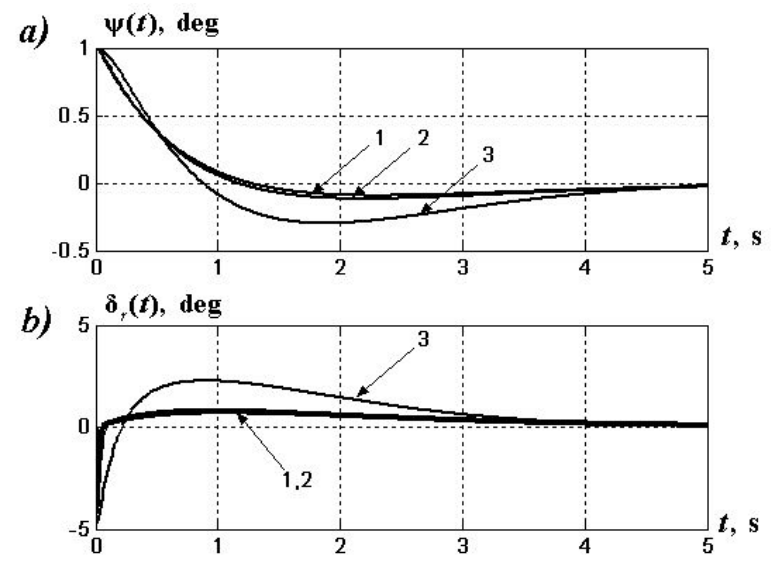

Figure 1: Yaw angle (a) and rudder deflection (b) time histories: 1) $h=0.1 \mathrm{~km}, 2$ ) $h=5 \mathrm{~km}, 3) h=9 \mathrm{~km}$.

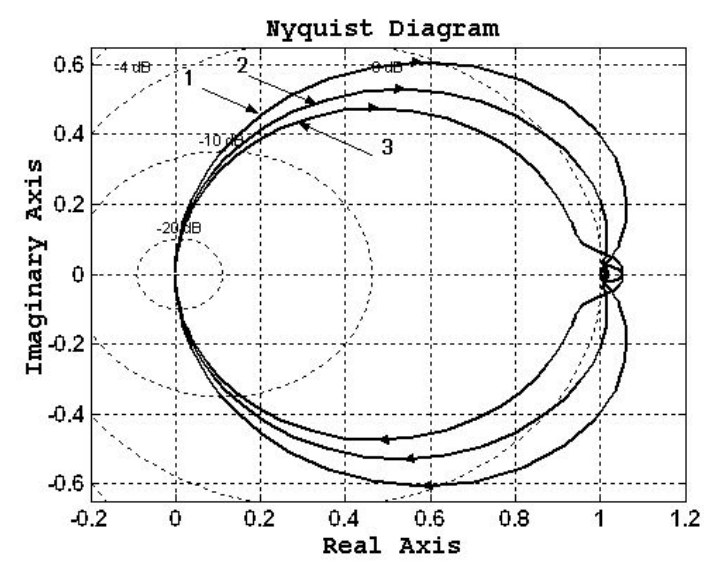

Figure 2: Nyquist plots: 1) $h=0.1 \mathrm{~km}, 2) h=5 \mathrm{~km}, 3) h=9 \mathrm{~km}$. 
All numerical calculations are performed in the MATLAB environment. YALMIP [14] is used to enter LMIs and semi-definite programming problems are solved with SeDuMi [22].

\section{Conclusions}

Non-conservative LMI conditions of robust strict G-passification are presented. For the quadratic passification case, a design method is given. All results are tested on an illustrative numerical example. Prospective work will be devoted to the design of robust strict $G$-passifying feedback control and to the more general problem of finding simultaneously a gain $G$ and the feedback $K$ that makes the closed-loop $G$-passive.

\section{Acknowledgments}

The authors wish to thank the Associate Editor and the anonymous reviewers for their helpful comments.

\section{References}

[1] B.R. Andrievsky, A.N. Churilov, and A.L. Fradkov. Feedback KalmanYakubovich lemma and its applications to adaptive control. In IEEE Conference on Decision and Control, pages 4537-4542, June 1996.

[2] G. Bianchini, A. Tesi, and A. Vicino. Synthesis of robust strictly positive real systems with $l_{2}$ parametric uncertainty. IEEE Trans. Circuits Systems I, 48(4):438-450, 2001.

[3] C.I. Byrnes, A. Isidori, and J.C. Willems. Passivity, feedback equivalence, and the global stabilization of minimum phase nonlinear systems. IEEE Trans. on Automat. Control, 36(11):1228-1240, 1991. 
[4] C.A.R. Crusius and A. Trofino. Sufficient LMI conditions for output feedback control problems. IEEE Trans. on Automat. Control, 44:1053-1057, 1999.

[5] A.L. Fradkov. Quadratic Lyapunov functions in the adaptive stabilization problem of a linear dynamic plant. Siberian Math. J., 2:341-348, 1976.

[6] A.L. Fradkov. Passification of non-square linear systems and feedback Yakubovich-Kalman-Popov lemma. European J. of Control, 6:573-582, 2003.

[7] A.L. Fradkov and B.R. Andrievsky. Shunting method for control of homing missiles with uncertain parameters. In IFAC Symposium on Automatic Control in Aerospace, volume 2, pages 33-38, St. Petersburg, Russia, June 2004.

[8] P. Gurfil. Robust guidance for electro-optical missiles. IEEE Trans. on AES, 30(2):450-461, 2003.

[9] K.M. Hangos, J. Bokor, and G. Szederkenyi. Analysis and Control of Nonlinear Process Systems. Springer, 2004.

[10] D. Henrion. Linear matrix inequalities for robust strictly positive real design. IEEE Trans. Circuits Systems - I, 49(7):1017-1020, 2002.

[11] C. Hollot and B. Barmish. Optimal quadratic stabilizability of uncertain linear systems. In 18th Allerton Conference on Communication and Computing, pages 697-706, University of Illinois, Monticello, 1980.

[12] A.G. Kelkar and S.M. Joshi. Robust control of non-passive systems via passification. In American Control Conference, pages 2657-2661, June 1997.

[13] P. Kokotovic and M. Arcak. Constructive nonlinear control: a historical perspective. Automatica, 37(5):637-662, 2001.

[14] J. Löfberg. YALMIP3, 2004. URL: http: / / control .ee.ethz . ch / joloef /yalmip.msql. 
[15] R. Lozano, B. Brogliato, O. Egeland, and B. Maschke. Dissipative systems analysis and control. Springer-Verlag London Ltd, 2000.

[16] R. Ortega, A. Loria, P.J. Nicklas son, and H. Sira-Ramirez. Passivity-based control of Euler-Lagrange systems. Springer-Verlag, Berlin, 1998.

[17] D. Peaucelle, D. Arzelier, and G.Garcia. Quadratic stabilisability and disk pole assignment for generalised uncertainty models - an LMI approach. In 2nd IMACS multiconference CESA, volume 1, pages 650-655, April 1998.

[18] D. Peaucelle, D. Henrion, and D. Arzelier. Quadratic separation for feedback connection of an uncertain matrix and an implicit linear transformation. In 16th IFAC World Congress, Prague, Czech Republic, July 2005. Submitted. LAAS-CNRS Tech. Report No 04435 also available at www . laas . fr / peaucell /papers /ifac05a.pdf.

[19] A. Saberi, P.V. Kokotović, and H.J. Sussmann. Global stabilization of partially linear composite systems. SIAM J.Contr. Optimiz., 28(6):1491-1503, 1990.

[20] R. Sepulchre, M. Janković, and P.V. Kokotović. Constructive nonlinear control. Springer-Verlag, New-York, 1996.

[21] M.M. Seron, D.J. Hill, and A.L. Fradkov. Adaptive passification of nonlinear systems. In IEEE Conference on Decision and Control, pages 190-195, December 1994.

[22] J.F. Sturm. Using SeDuMi 1.02, a MATLAB toolbox for optimization over symmetric cones. Optimization Methods and Software, 11-12:625-653, 1999. URL: fewcal.kub.nl / sturm/software/sedumi.html.

[23] W. Sun, P. Khargonekar, and D. Shim. Solution to the positive real control problem for linear time-invariant systems. IEEE Trans. on Automat. Control, 39(10):2034-2046, 1994.

[24] L. Xie, M. Fu, and H. Li. Passivity analysis and passification for uncertain signal processing systems. IEEE Trans. Signal Processing, 46(9):23942403, 1998. 
[25] B. E. Ydstie. Passivity based control via the second law. Computers \& Chemical Engineering, 26(7-8):1037-1048, 2002. 